\title{
Gamma-Aminobutyric Acid
}

National Cancer Institute

\section{Source}

National Cancer Institute. Gamma-Aminobutyric Acid. NCI Thesaurus. Code C80523.

A naturally occurring neurotransmitter with central nervous system (CNS) inhibitory activity. Gamma-aminobutyric acid (GABA), converted from the principal excitatory neurotransmitter glutamate in the brain, plays a role in regulating neuronal excitability by binding to its receptors, GABA-A and GABA-B, and thereby causing ion channel opening, hyperpolarization and eventually inhibition of neurotransmission. 